\title{
Proton pump inhibitor-induced hypomagnesaemia leading to recurrent falls and delirium: a case report
}

Tanya Joy Zapata Quicho, MD, GDGRM, Christine Yuanxin Chen, MBBS, MRCP

\begin{abstract}
Older adults are at increased risk of developing hypomagnesaemia secondary to chronic use of proton pump inhibitors. We report a 73-yearold woman who presented with acute functional decline, recurrent falls, and cognitive impairment likely owing to unrecognised persistent hypomagnesaemia secondary to use of proton pump inhibitors.
\end{abstract}

Key words: Aged; Proton pump inhibitors

\section{CASE REPORT}

\begin{abstract}
Department of Geriatric Medicine, Changi General Hospital, Singapore
\end{abstract}

Correspondence to: Tanya Joy Zapata Quicho, Department of Geriatric Medicine, Changi General Hospital, Singapore. Email: Quicho_Tanya@cgh.com.sg

\section{INTRODUCTION}

Proton pump inhibitors have been reported to cause hypomagnesaemia. Manifestations of hypomagnesaemia include muscle weakness, tremors, delirium, coma and even death. We present a 73-year-old woman who had progressive muscle weakness, recurrent falls, arrhythmias, and delirium secondary to hypomagnesaemia.

\section{CASE PRESENTATION}

In February 2020, a 73-year-old Chinese woman with comorbidities of hypertension, type II diabetes mellitus, hyperlipidaemia, and ischaemic heart disease was admitted to the acute geriatric ward with sudden unresponsiveness during a meal.

Before October 2019, the patient could walk independently. However, she started to have recurrent falls and was hospitalised in late October 2019. Since then, she had an acute decline in function and cognition. She was unable to walk and needed moderate to maximum assistance in transfers. She also became less conversant and had a decline in cognitive function. It was noted that her magnesium levels were persistently low at 0.4 to $0.5 \mathrm{mmol} / \mathrm{L}$.

Upon this current admission, she had irregular heart sounds and weakness of all four limbs. She was not able to stand independently. She could not complete the abbreviated mental test, as she was not responding consistently. She was found to have a stage 3 sacral pressure injury.

Magnetic resonance imaging result of the brain was unremarkable. Blood test results showed hypernatraemia $(150 \mathrm{mmol} / \mathrm{L})$, hypokaelaemia $(2.5 \mathrm{mmol} / \mathrm{L})$, and hypomagnaesemia $(0.19 \mathrm{mmol} / \mathrm{L})$. She had no hypoglycaemia or anaemia and her blood pressure was normal. Inflammatory markers were normal. Electrocardiography showed frequent premature ventricular complexes. A 24-hour Holter showed some atrial ectopics and infrequent ventricular ectopics. Transthoracic echocardiography showed an ejection fraction of $55 \%$ with no wall motion abnormalities or valvular disease.

She had dysphagia and was placed on a modified diet. On transfer to the subacute ward, she was drowsy. She could open her eyes to calling but was not orientated and could not sustain attention. The power of all four limbs was $2 / 5$. Her sitting balance was very poor and there was no neck control.

Repeat blood tests revealed that her magnesium levels dropped to $0.33 \mathrm{mmol} / \mathrm{L}$. This was replenished intravenously. A drug review showed that one of her chronic medications was omeprazole, which was stopped while magnesium was being replenished. 
Her thyroid and parathyroid hormone levels and serum calcium and potassium levels were normal. The magnesium levels recurrently dropped and several courses of magnesium replenishment were given. After intravenous and oral replenishment and maintenance magnesium tablets, her serum magnesium level normalised to around $0.7 \mathrm{mmol} / \mathrm{L}$, and her attention span improved. She scored 5/10 on the abbreviated mental test. She recovered some sitting balance, and the power in all limbs improved to at least $3 / 5$.

The renal specialist concurred that the hypomagnesaemia was most likely caused by omeprazole. Omeprazole was stopped permanently, and she was maintained on oral magnesium. At the home visit 1 month post discharge, her magnesium level was normal. She was followed up for 3 months and had no further improvement in cognition. She was subsequently diagnosed with dementia.

\section{DISCUSSION}

Proton pump inhibitor-induced hypomagnesaemia may result from impaired intestinal absorption owing to reduced active transport of the $\mathrm{Mg}^{2+}$ in the reduced intraluminal $\mathrm{pH} .{ }^{1}$ The reported time to development of hypomagnesaemia ranges from 14 days to 13 years. ${ }^{2}$ Older adults with multiple comorbidities (diabetes mellitus, polypharmacy, and poor dietary intake) are at higher risk of developing hypomagnesaemia.

When older adults present with falls and loss of consciousness, initial management is to rule out cardiac and neurologic pathologies. Hypomagnesaemia is usually not considered a cause of cognitive impairment or delirium. Our case illustrates the importance of including testing of serum levels of magnesium in patients with falls and functional decline. Serum levels of magnesium should be monitored frequently during treatment of hypomagnesaemia, as magnesium is an intracellular ion, and its repletion may not be reflected correctly on the first normal level result. Repeat testing is thus important.

It is imperative to evaluate the underlying cause of hypomagnesaemia to prevent recurrence. ${ }^{3}$ Proton pump inhibitor use of more than a year significantly increases the risk of hypomagnesaemia. ${ }^{4,5}$ As a large population of older patients are prescribed with proton pump inhibitors indefinitely, medication review for appropriateness of long-term proton pump inhibitor use should be routinely performed. Serum magnesium levels should be checked before and during proton pump inhibitor therapy, or when there are acute changes in function or cognition. This may prevent prolonged state of hypomagnesaemia and its complications.

\section{CONTRIBUTORS}

All authors designed the study, acquired the data, analysed the data, drafted the manuscript, and critically revised the manuscript for important intellectual content. All authors had full access to the data, contributed to the study, approved the final version for publication, and take responsibility for its accuracy and integrity.

\section{CONFLICTS OF INTEREST}

All authors have disclosed no conflicts of interest.

\section{FUNDING/SUPPORT}

This study received no specific grant from any funding agency in the public, commercial, or notfor-profit sectors.

\section{ETHICS APPROVAL}

The patient was treated in accordance with the tenets of the Declaration of Helsinki. The patient provided written informed consent for all treatments and procedures.

\section{REFERENCES}

1. Park $\mathrm{CH}$, Kim EH, Roh YH, Kim HY, Lee SK. The association between the use of proton pump inhibitors and the risk of hypomagnesemia: a systematic review and meta-analysis. PLoS One 2014;9:e112558. Crossref

2. Koh ZY, Lim SWM, Lim WS. An interesting case of "refractory" hypomagnesemia. J Am Med Dir Assoc 2017;18:632-3. Crossref

3. Martin KJ, Gonzalez EA, Slatopolsky E. Clinical consequences and management of hypomagnesemia. J Am Soc Nephrol 2009;20:2291-5. Crossref

4. Hoorn EJ, van der Hoek J, de Man RA, Kuipers EJ, Bolwerk C, Zietse R.A case series of proton pump-induced hypomagnesemia. Am J Kidney Dis 2010;56:112-6. Crossref

5. Kim S, Lee H, Park $\mathrm{CH}$, et al. Clinical predictors associated with proton pump inhibitor-induced hypomagnesemia. Am J Ther 2015;22:14-21. Crossref 